\title{
Mutagenic and inhibitory compounds produced by fungi affect detrimentally diagnosis and phylogenetic analyses.
}

\begin{abstract}
Microorganisms manufacture prolifically bioactive compounds. For example, fungi produce antibiotics and mycotoxins. However, many are difficult to identify and classify. Methods which rely on nucleic acid (DNA/RNA) are increasingly being used for this purpose where strains are grown in liquid or agar culture and often subjected to polymerase chain reaction (PCR) analyses. It has not been considered that self-produced mutagenic and inhibitory secondary metabolites (SM) affect DNA analysis of the target fungi. The most obvious mycotoxins and fungi to consider in this regard are aflatoxins (AFB) and Aspergillus, as AFB are the most mutagenic natural compounds. Many other fungi and SM are relevant and fungi act as a model for bacteria and plants. In fact, fungi repair damaged nucleic acid (NA) and are capable of removing toxins by employing transporter proteins. Nevertheless, these could be inhibited by bioactive metabolites. Mutagenic effects may involve inhibition of DNA stabilising enzymes. In addition, PCR is subject to false negative results. Samples of fungi with the genes of interest (e.g. a mycotoxin) may be categorized as negative and safe as a consequence. Internal amplification controls (IACs) will ameliorate the situation and need to become mandatory. These are conventionally NA that posses a sequence which will provide a PCR product (a) using the same primers employed for the target gene and (b) that will not coincide on the gel with the product of the target gene. Inhibitors and mutagens in cultures need to be minimized, and SM are an obvious source. This is a crucial issue in developing diagnostic and phylogenetic methods. The conclusions are (a) previous reports are compromised because IACs have not been employed in PCR and (b) mutagens and inhibitors may affect the very stability essential for NA analyses used in diagnostics and phylogenetics.
\end{abstract}

Keyword: Inhibitory compounds; Mutagenic; Fungi affect; Phylogenetic analyses; Microorganisms; Bioactive compounds; Polymerase chain reaction (PCR); Secondary metabolites (SM); Aspergillus 